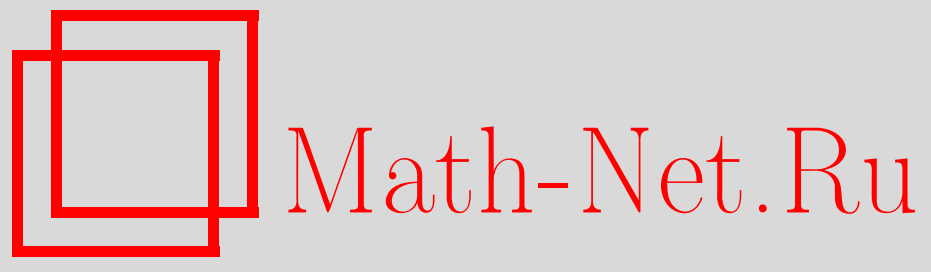

Ю. М. Устиновский, Операция удвоения многогранников и действия тора, УМH, 2009, том 64, выпуск 5, 181182

DOI: https://doi.org/10.4213/rm9301

Использование Общероссийского математического портала Math-Net.Ru подразумевает, что вы прочитали и согласны с пользовательским соглашением http://www . mathnet.ru/rus/agreement

Параметры загрузки:

IP : 54.164 .48 .24

26 апреля 2023 г., 12:58:57

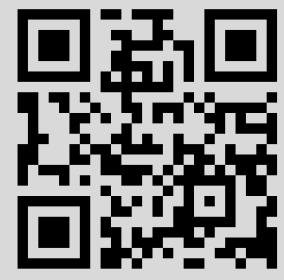




\section{Операция удвоения многогранников и действия тора}

\section{Ю. М. Устиновский}

В заметке определяется операция удвоения на простых многогранниках и приводится формула, связывающая $h$-полиномы исходного и нового многогранников. В качестве примера применения этой операции дается доказательство гипотезы о торическом ранге для момент-угол-многообразий $Z_{P}$. Для доказательства последней мы устанавливаем связь между момент-угол-многообразиями и их вещественными аналогами, здесь нам и понадобится операция удвоения.

Пусть $n$-мерный простой многогранник $P$ с $m$ гипергранями задан системой в $\mathbb{R}^{n}$ :

$$
\left(x, a_{i}\right)+b_{i} \geqslant 0, \quad x, a_{i} \in \mathbb{R}^{n}, \quad i=1, \ldots, m .
$$

В таком случае $P$ отождествляется с пересечением положительного ортанта $\mathbb{R}_{\geqslant}^{m}$ и образа вложения $i_{P}: \mathbb{R}^{n} \rightarrow \mathbb{R}^{m}, i_{P}(x)=A_{P}(x)+b_{P}$, где $A_{P}$ - матрица размера $m \times n$, по строкам которой записаны векторы $a_{i}$, а $b_{P}-$ вектор-столбец из $b_{i}$. Зададим образ вложения $i_{P}$ системой $m-n$ уравнений в $\mathbb{R}^{m}$ :

$$
\sum_{i=1}^{m} c_{j, i} x_{i}=q_{j}, \quad j=1, \ldots, m-n .
$$

ОПРеДЕЛЕНИЕ 1. Удвоением многогранника $P$ называется многогранник $L(P)$, заданный системой $m-n$ уравнений в $\mathbb{R}_{\geqslant}^{2 m}$ :

$$
\sum_{i=1}^{m} c_{j, i}\left(x_{i}+x_{i}^{\prime}\right)=q_{j}, \quad j=1, \ldots, m-n .
$$

Заметим, что $L(P)$ есть $(m+n)$-мерный простой многогранник с $2 m$ гипергранями, $L(P \times Q)=L(P) \times L(Q)$. Если $\left\{v_{1}, \ldots, v_{m}\right\}$ - диаграмма Гейла многогранника $P$ (см. [1]), то $\left\{v_{1}, v_{1}, \ldots, v_{m}, v_{m}\right\}$ - диаграмма Гейла многогранника $L(P)$.

Пусть $f_{n-i, i}$ - число граней многогранника $P$ размерности $n-i$. Тогда $h$-полиномом многогранника $P$ называется полином, определяемый соотношением $h(P)(\alpha, t)=$ $f(\alpha-t, \alpha)$, где $f(P)(\alpha, t)=\alpha^{n}+f_{n-1,1} \alpha^{n-1} t+\cdots+f_{0, n} t^{n}$.

Лemma 2. $h(L(P))(\alpha, t)=\sum(-1)^{\operatorname{codim} G}(\alpha t)^{\operatorname{codim} G}(\alpha+t)^{m-\operatorname{codim} G} h(G)(\alpha, t)$, гдe суммирование ведется по всем граням $P$, включая сам многогранник.

Пусть $(\mathscr{P}, d)$ - дифференциальное кольцо простых многогранников [2]. Умножение в $\mathscr{P}$ задается произведением многогранников, сложение формально, а дифференциал задается формулой $d(P)=\sum F_{i}$, где в правой части стоит сумма гиперграней многогранника $P$. Пусть $h: \mathscr{P} \rightarrow \mathbb{Z}[\alpha, t]$ - гомоморфизм, определяемый $h$-полиномом, тогда $h(d(P))(\alpha, t)=\left(\partial_{\alpha}+\partial_{t}\right) h(P)(\alpha, t)$ [2]. Это позволяет переформулировать лемму 2 следующим образом:

$$
h(L(P))(\alpha, t)=(\alpha+t)^{m}\left(\sum_{i=0}^{\infty} \frac{(-1)^{i}}{i !}(\alpha t)^{i}(\alpha+t)^{-i}\left(\partial_{\alpha}+\partial_{t}\right)^{i}\right) h(P)(\alpha, t) .
$$

Дифференциальный оператор, заключенный в скобки, мультипликативен, поэтому, вычислив его значение на $\alpha$ и $t$, мы получаем следующее утверждение.

Teоpema 3. $h(L(P))(\alpha, t)=(\alpha+t)^{m-n} h(P)\left(\alpha^{2}, t^{2}\right)$.

Теперь перейдем к основному для нас примеру применения операции удвоения.

ОПределение 4 . Пусть $\mu: \mathbb{C}^{m} \rightarrow \mathbb{R}_{\geqslant}^{m}, \mu\left(z_{1}, \ldots, z_{m}\right)=\left(\left|z_{1}\right|^{2}, \ldots,\left|z_{m}\right|^{2}\right),-$ проекция на пространство орбит стандартного действия тора $T^{m}$. Момент-угол-многообразием $Z_{P}$ называется множество $\mu^{-1}\left(i_{P}(P)\right)$ (см. [3]). 
ОПределение 5 . Пусть $\pi: \mathbb{R}^{m} \rightarrow \mathbb{R}_{\geqslant}^{m}, \pi\left(x_{1}, \ldots, x_{m}\right)=\left(x_{1}^{2}, \ldots, x_{m}^{2}\right),-$ проекция на пространство орбит стандартного действия группы $\mathbb{Z}_{2}^{m}$. Вещественным моментугол-многообразием $R_{P}$ называется множество $\pi^{-1}\left(i_{P}(P)\right)$.

ГИПОТЕЗА О ТОРИЧЕСКОМ РАНГЕ [4]. Если тор $T^{r}$ действует почти свободно на конечномерном пространстве $X$, mо $\operatorname{hrk}(X) \geqslant 2^{r}$, где $\operatorname{hrk}(X)=\sum_{i} \operatorname{dim} H^{i}(X)$.

Простота многогранника $P$ влечет наличие на $Z_{P}$ и на $R_{P}$ структуры гладкого многообразия, [3]. На многообразиях $Z_{P}$ имеется естественное действие тора $T^{m}$, при этом ранг подтора, действующего на $Z_{P}$ почти свободно, равен $m-n$ (см. [5]). Мы покажем, что $\operatorname{hrk}\left(Z_{P}\right) \geqslant 2^{m-n}$, тем самым, гипотеза о торическом ранге выполняется для момент-угол многообразий.

Лемма 6. Всякое многообразие $Z_{P}$ гомеоморфно многообразию $R_{L(P)}$.

ДокАЗАтЕльство. Пусть многогранник $P$ задан системой вида (1). Тогда из определений следует, что многообразие $Z_{P}$ может быть задано системой уравнений в $\mathbb{C}^{m}$ : $\sum_{i=1}^{m} c_{j, i}\left|z_{i}\right|^{2}=q_{j}, j=1, \ldots, m-n$.

$\mathrm{B}$ вещественных координатах $z_{i}=x_{i}+\sqrt{-1} y_{i}$ мы получим систему уравнений $\sum_{i=1}^{m} c_{j, i}\left(x_{i}^{2}+y_{i}^{2}\right)=q_{j}, j=1, \ldots, m-n$, задающую многообразие $R_{L(P)}$ в $\mathbb{R}^{2 m}$.

Теорема 7. Для когомологий всякого вещественного момент-угол-многообразия $R_{P}$ справедливо неравенство $\mathrm{hrk}\left(R_{P}\right) \geqslant 2^{m-n}$.

Следующая лемма доказывается применением точной последовательности Майера-Вьеториса.

Лемма 8. Пусть $M$ - компактное многообразие с краем, а многообразие $X$ получается склейкой двух копий $M$ вдоль общей гранищь. Тогда $\operatorname{hrk}(X) \geqslant \operatorname{hrk}(\partial M)$.

ДокАЗАТЕЛЬСтво теоРемы 7. Пусть утверждение доказано для многогранников размерности $n-1$, а $P$ - произвольный $n$-мерный простой многогранник. Обозначив $M=\left\{\vec{x} \in R_{P} \mid x_{1} \geqslant 0\right\}$, получаем, что $R_{P}$ есть склейка двух экземпляров многообразия $M$ вдоль границы: $R_{P}=M \cup_{\partial M} M$. Поэтому, по лемме $8, \operatorname{hrk}\left(R_{P}\right) \geqslant$ $\operatorname{hrk}(\partial M)$. Нетрудно видеть, что $\partial M=\pi^{-1}\left(i_{P}\left(F_{1}\right)\right)=R_{F_{1}} \times \mathbb{Z}_{2}^{k}$, где $F_{1}$ - гипергрань, задаваемая обращением $x_{1}$ в 0 , а $k$ - число гиперграней $F$ многогранника $P$ таких, что $F_{1} \cap F=\varnothing$. По предположению индукции для $\partial M$ имеем: $\operatorname{hrk}(\partial M)=2^{k} \operatorname{hrk}\left(R_{F_{1}}\right) \geqslant$ $2^{k} \cdot 2^{(m-k-1)-(n-1)}$. Откуда $\operatorname{hrk}\left(R_{P}\right) \geqslant \operatorname{hrk}(\partial M) \geqslant 2^{m-n}$, что и требовалось.

С учетом доказанной теоремы и леммы 6 мы получаем следующее неравенство:

$$
\operatorname{hrk}\left(Z_{P}\right)=\operatorname{hrk}\left(R_{L(P)}\right) \geqslant 2^{2 m-(m+n)}=2^{m-n},
$$

влекущее утверждение гипотезы о торическом ранге для момент-угол-многообразий.

Имеется обобщение операции удвоения для произвольных симплициальных комплексов $K$, которое позволяет доказать гипотезу о торическом ранге для произвольных момент-угол-комплексов $Z_{K}$; необходимые определения можно найти в [5].

Автор выражает благодарность Т. Е. Панову и С. Гитлеру (Samuel Gitler) за привлечение внимания к операции удвоения многогранников, а также В. М. Бухштаберу и научному руководителю Т. Е. Панову за постановку задачи и внимание к работе.

\section{Список литературы}

[1] G. M. Ziegler, Lectures on polytopes, Grad. Texts in Math., 152, Springer, Berlin, 1995. [2] B. М. Бухштабер, Tp. МИАН, 263 (2008), 1-26. [3] V. M. Buchstaber, T. E. Panov, N. Ray, Mosc. Math. J., 7:2 (2007), 219-242. [4] S. Halperin, London Math. Soc. Lecture Notes, 93, 1985, 293-306. [5] В. М. Бухштабер, Т. Е. Панов, Тр. МИАН, 247 (2004), 41-58.

Ю. М. Устиновский (Yu. M. Ustinovskii)

Московский государственный университет им. М. В. Ломоносова

E-mail: yura.ust@gmail.com
Представлено В. М. Бухштабером Принято редколлегией 16.06.2009 